\title{
HARRISON PRIMES IN A RING WITH FEW ZERO DIVISORS
}

\author{
MAX D. LARSEN ${ }^{1}$
}

1. Introduction. In [4] D. K. Harrison introduces preprimes, primes, and valuation preprimes to pave the way for an algebraic number theory in arbitrary commutative rings. He shows $[4, \S 3]$ that his definitions coincide with the classic definitions of valuation theory when restricted to fields. In this paper the author extends Harrison's results to a large class of rings which includes all Noetherian rings. In addition an analog to the approximation theorem for valuations is developed for this class of rings.

Let $R$ be a commutative ring with unity consisting entirely of zero divisors and units and having finitely-many maximal ideals, for example, the total quotient ring of a Noetherian ring. The adjunction of the word "regular" to a nonempty subset of $R$ will mean that the set contains at least one regular element; i.e., a nonzero divisor. Let $X$ be the set of regular finite primes $P$ of $R$ such that the total quotient ring of $P: P$ is $R$. Then all these subrings have few zero divisors $[3$, p. 203]. The terminology and results of [3] and [4] will be used freely. For any subring $A$ of $R$ and multiplicatively-closed subset $S$ of $A$ consisting of regular elements, we will assume that the ring of quotients $A_{S}$ is canonically embedded in $R$. For two subrings $A, B$ of $R, A: B=\{x \in R \mid x B \subseteq A\}$. In placing the above restriction on $R$ and $X$, the author is prescribing a situation resembling the situation involved in the classical valuation theory in fields.

Give $X$ the topology generated by taking as basic open sets all sets of the form $\{P \in X \mid P \cap E=\varnothing\}$ where $E$ is a finite set of regular elements of $R$. This is not the same topology as Harrison uses in [4, p. 10]. Let $X^{\#}$ be the set of closed irreducible subsets $C$ of $X$ such that $\cap C=\cap\{P \mid P \in C\}$ is regular. Give $X^{\sharp}$ the topology which takes sets of the form $\{C \in X \mid C \cap V \neq \varnothing\}$, for some open subset $V$ of $X$, as open sets. Let $\mathfrak{X}$ be the set of all regular valuation preprimes $S$ of $R$ such that the total quotient ring of $S: S$ is $R$ and topologize $\mathfrak{X}$ by using $\{S \in \mathfrak{X} \mid S \cap E=\varnothing\}$, for a finite set $E$ of regular elements of $R$, as basic open sets. A regular valuation pair $(A, P)$ of $R$ is simply a valuation pair $(A, P)$ with $P$ regular.

Received by the editors August 29, 1968.

1 During the writing of this paper the author received support from NSF under Grant GP-8932. 


\section{Extension of Harrison's results.}

TheORem 1. Let $P$ be a regular preprime of $R$ and let $A=P: P$. $P$ is a valuation preprime of $R$ with $R$ as the tolal quotient ring of $A$ if and only if $(A, P)$ is a valuation pair of $R$.

The proof of this assertion is embodied in the following propositions which will show that $X^{\sharp}$ and $X$ are homeomorphic under the maps $C \in X^{\sharp} \rightarrow \cap C$ and $P \in \mathfrak{X} \rightarrow\{Q \in X \mid P \subseteq Q\}$. The continuity of these maps is easy to check after the propositions establish their one-oneness.

REMARKs. (1) The definition of certain valuation pairs can be weakened. Let $(A, P)$ be such that $A$ is a subring of $R$ whose total quotient ring is $R, P$ a regular prime ideal of $A$, and for all regular $x \in R \backslash A$, there exists $y \in P$ such that $x y \in A \backslash P$. Then $(A, P)$ is a valuation pair of $R$, for if $z \in R \backslash A$, then there exists $w \in P$ such that $z+w$ is regular. Then $z+w \in R \backslash A$ implies the existence of $y \in P$ such that $(z+w) y \in A \backslash P$. Hence $z y \in A \backslash P$. The existence of the $w \in P$ such that $z+w$ is regular follows by an argument similar to that used by Davis [3, Lemma B, p. 204] and using the fact that $P$ is a regular ideal of $A$.

(2) A similar result is true for regular valuation preprimes. Let $S$ be a regular preprime such that $-S \subseteq S$, the total quotient ring of $S: S$ is $R$, and for any finite set $E$ of regular elements of $R$ such that $S \cap E=\varnothing$, there exists a finite prime $P$ of $R$ such that $S \subseteq P$ and $P \cap E=\varnothing$. Let $E=\left\{e_{1}, \cdots, e_{n}\right\}$ be any subset of $R$. Then there exists $u_{1}, \cdots, u_{n} \in S$ such that $\left\{e_{1}+u_{1}, \cdots, e_{n}+u_{n}\right\}$ is a set of regular elements disjoint from $S$. Hence there exists a finite prime $P$ such that $S \subseteq P$ and $e_{i}+u_{i} \notin P$ for $i=1, \cdots, n$. But then $e_{i} \notin P$ for $i=1, \cdots, n$, so $S$ is a valuation preprime.

(3) The ordering on the set of all pairs $(A, P)$ where $A$ is a subring of $R$ and $P$ a prime ideal of $A$ is $\left(A_{1}, P_{1}\right) \leqq\left(A_{2}, P_{2}\right)$ if and only if $A_{1} \subseteq A_{2}$ and $P_{1}=P_{2} \cap A_{1}$. Manis [6, Definition 1.8] has established that maximal pairs and valuation pairs of $R$ are equivalent terms. For any regular valuation pair $(A, P)$ of $R$, we have $(A, P)$ $\leqq\left(A_{S(P)}, P A_{S(P)}\right)$ where $S(P)$ denotes the set of regular elements in the complement of $P$ in $A$. Hence by the maximality of $(A, P), P$ must be the unique maximal regular ideal of $A$ [3, Lemma A, p. 204].

(4) Let $(A, P)$ be a valuation pair of $R$. For a regular element $x \in R$, if $x, x^{-1} \in R \backslash A$, then there exists $y, z \in P$ such that $x y, x^{-1} z$ $\in A \backslash P$. Thus $y z \in P \cap(A \backslash P)$ which is impossible. Thus $x$ or $x^{-1}$ must be in $A$. Consequently when $(A, P)$ is a valuation pair of $R, A$ is a quasi-valuation ring [3, p. 203]. On the other hand, if $A$ is a quasivaluation ring and $P$ is its unique maximal regular ideal, then Remark 1 shows that $(A, P)$ is a regular valuation pair of $R$. 
(5) Remark 4 and [3, Proposition 5, p. 205] imply that if $(A, P)$ is a valuation pair of $R$ and $(B, Q)$ is a pair where $B$ is a subring of $R$ containing $A$ and $Q$ is the maximal regular ideal of $B$, then $(B, Q)$ is a valuation pair of $R$ and $B=A_{S(Q)}$.

Proposition 1. Let $C \in X^{\sharp}$ with $P=\cap C$ and $A=P: P$. Then $(A, P)$ is a valuation pair of $R$.

Proof. Let $p$ be a regular element of $P$. Clearly $A$ is a subring of $R$ and $P$ is an ideal of $A$. Suppose $a, b \in A$ with $a \notin P, b \notin P$, and $a b \in P$. Then there exist $u, v \in A$ such that $c=a+u p, d=b+v p$ are regular and $c \notin P, d \notin P$, and $c d \in P$. Hence $\{Q \in C \mid c \notin Q\}$ and $\{Q \in C \mid d \notin Q\}$ are nonempty open subsets of $C$ whose intersection is empty contrary to the irreducibility of $C$. Thus $P$ is a prime ideal of $A$.

Let $x \in R \backslash A, x$ regular. Then since $x \notin A$ there exists $y \in P$ such that $x y \notin P$. There exists $u \in A$ such that $y+u p$ is regular and $u \in(A: x)$. [This is Davis' argument again using the fact that $A \cap(A: x)$ is a regular ideal of $A$.] Then $y+u p=x^{-1}(x(y+u p)) \in P$ and $x(y+u p) \notin P$. An argument similar to the one above based on the irreducibility of $C$ shows that the complement of $P$ in $R$ is closed under multiplication of regular elements. Hence $x^{-1} \in P$. Hence for $x$ regular in $R \backslash A$, there exists $x^{-1} \in P$ such that $x x^{-1} \in A \backslash P$. By $\mathrm{Re}-$ mark 1 this proves that $(A, P)$ is a valuation pair of $R$. Note that $x^{-1} \in A$ for all regular $x \in R \backslash A$ implies that the total quotient ring of $A$ is $R$.

Proposition 2. If $(A, P)$ is a valuation pair of $R$, then $P$ is a valuation preprime of $R$.

Proof. Let $E$ be a finite set with $P \cap E=\varnothing$. Let $E=E_{1} \cap E_{2}$ where $E_{1}=\left\{e_{1}, \cdots, e_{s}\right\} \subseteq A$ and $E_{2}=\left\{e_{s+1}, \cdots, e_{r}\right\} \subseteq R \backslash A$. For $i=s+1, \cdots, r$, there exist $t_{i} \in P$ such that $e_{i} t_{i} \in A \backslash P$. Let $E^{\prime}$ $=\left\{e_{1}, \cdots, e_{s}, e_{s+1} t_{s+1}, \cdots, e_{r} t_{r}\right\}$. Then $E^{\prime} \subseteq A$ so $E^{\prime} P \subseteq P$. By [4, Lemma 2.6, p. 21], there exists a finite prime $Q$ of $R$ such that $P \subseteq Q$ and $Q \cap E^{\prime}=\varnothing$. Thus $E_{1} \cap Q=\varnothing$ and for $i=s+1, \cdots, r, e_{i} t_{i} \in Q$ and $t_{i} \in Q$ imply that $e_{i} \notin Q$ so $E_{2} \cap Q=\varnothing$. Thus $E \cap Q=\varnothing$.

For a valuation preprime $P$ of $R$, let $C_{P}=\{Q \in X \mid P \subseteq Q\}$.

Proposition 3. If $P$ is a valuation preprime of $R$ and $\cap C_{P}$ is regular, then $C_{P}$ is a closed irreducible subset of $X$.

Proof. The irreducibility follows readily. To see that $C_{P}$ is closed note that $C_{P}$ is the complement of

$$
U\{Q \in X \mid\{x\} \cap Q=\varnothing \quad \text { for some regular } x \in P\} .
$$

One inclusion is clear; for the other, note that for $Q \notin C_{P}$, there exists 
$x \in P, x \notin Q$. If $p$ is a regular element of $\cap C_{P}$, then there is a $u \in P: P$ such that $x+u p$ is regular, $x+u p \in P, x+u p \notin Q$. Therefore $Q$ is not in the complement mentioned above.

Proposition 4. If $P$ is a regular valuation preprime of $R$, then $P=\cap C_{P}$.

Proof. Clearly $P \subseteq \cap C_{P}$. Let $x \in R \backslash P$ and let $A=P: P$. If $x \in A \backslash P$, then there exists $Q \in C_{P}$ such that $x \notin Q$; hence $x \notin \cap C_{P}$. On the other hand, $x \in R \backslash A$. By Proposition $3 C_{P}$ is a closed irreducible subset of $X$. Thus by Proposition $1(A, P)$ is a valuation pair of $R$. Hence there exists $y \in P$ such that $x y \in A \backslash P$. Hence there exists $Q \in C_{P}$ such that $x y \notin Q$. But $y \in P \subseteq Q$ implies $x \notin Q$. Hence $x \notin \cap C_{P}$.

3. Relationship to integral closure. In this section we show that the integral closure of a ring has the same characterization as in the classical setting. First a lemma.

Lemma 1. Let $T$ be a subring of $R, I$ a proper regular ideal of $T$. Then there exists a valuation pair $(A, P)$ of $R$ such that $I \subseteq P$.

Proof. Consider the set of all pairs $(A, P)$ where $A$ is a subring of $R$ and $P$ is a prime ideal of $A$ containing $I$. This inherits the ordering discussed in Remark 3 and in this ordering has a maximal element which is also maximal in the set of all such pairs with no restriction regarding $I$. Hence this maximal element is a valuation pair satisfying the assertion of the lemma.

THEOREM 2. For any subring $A$ of $R$ whose total quotient ring is $R$, the integral closure $\bar{A}$ of $A$ is

$$
A^{*}=\bigcap_{\alpha}\left\{A_{\alpha} \mid\left(A_{\alpha}, P_{\alpha}\right) \text { is a valuation pair of } R, A \subseteq A_{\alpha}\right\} \text {. }
$$

Proof. $\bar{A} \subseteq A^{*}$ since each $A_{\alpha}$ is integrally closed [6, Proposition 1.9]. Now let $x \in R \backslash \bar{A}, x$ regular. Then $x^{-1}$ is not a unit in $A\left[x^{-1}\right]$, for if it were, then $x=a_{0}+a_{1} x^{-1}+\cdots+a_{s} x^{-s}$ for $a_{0}, a_{1}, \cdots, a_{s} \in A$ which would violate $x \notin \bar{A}$. Thus $x^{-1} A\left[x^{-1}\right]$ is a proper ideal of $A\left[x^{-1}\right]$. Thus, by Lemma 1 , there exists a valuation pair $(B, P)$ of $R$ such that $x^{-1} A\left[x^{-1}\right] \subseteq P$. Since $x^{-1} \in P, x \in B$. Thus the regular elements of $A^{*}$ belong to $A$. Hence by [3, Lemma C, p. 205] $A^{*} \subseteq \bar{A}$.

4. An approximation theorem for valuations. The following definitions and results are due to Manis [6, Proposition 1.6]. Let $(A, P)$ be a valuation pair of $R$. For $x, y \in R$, set $x \sim y$ if and only if $P:(x)$ $=P:(y)$. Let $V(x)=\{y \in R \mid y \sim x\}$ and $\Gamma_{V}=\{V(x) \mid x \in R\}$. Then $\Gamma_{V} \backslash V(0)$ is a group with identity $V(1)=A \backslash P$ and composition 
$V(x) V(y)=V(x y)$ for all $x, y \in R$. Define $V(x)<V(y)$ if there exists $z \in R$ such that $x z \in P$ and $y z \in A \backslash P$. Then $<$ is a linear order on $\Gamma_{V}, \Gamma_{V} \backslash V(0)$ is an ordered group, and $V(x+y) \leqq \max \{V(x), V(y)\}$ for all $x, y \in R$.

We use these facts to prove an approximation theorem for valuations. The approach here imitates that of [2, p. 132-136]. Again, if $P$ is a prime ideal of a ring $A, S(P)$ denotes the set of regular elements in the complement of $P$ in $A$. Using the previous remarks, [5, Lemma 3.1] may be restated as

Theorem 3. Let $\left\{\left(A_{i}, P_{i}\right)\right\}_{i=1, \cdots, n}$ be a set of regular valuation pairs of $R$. Let $A=\bigcap_{i=1}^{n} A_{i}$ and set $Q_{i}=P_{i} \cap A$ for $i=1, \cdots, n$. Then $A_{i}=$ $A_{S\left(Q_{i}\right)}$ for $i=1, \cdots, n$ and if $A_{i} \Phi A_{j}$ for $i \neq j$, then $\left\{Q_{i}\right\}_{i=1, \cdots, n}$ is the set of maximal regular ideals of $A$.

Lemma 2. Let $(A, P)$ be a valuation pair of $R, M$ a proper regular ideal of $A$, and $N=\sqrt{ } M$. Then $N$ is a prime ideal of $A$.

Proof. Let $V$ be the valuation defined by $(A, P)$ and let $m$ be a regular element of $M$. Let $x, y \in A$ with $x y \in N$. There exist $u, v \in A$ such that $s=x+u m, t=y+v m$ are regular. Then $s t \in N$ so $(s t)^{n} \in M$ for some positive integer $n$. If $V(s) \geqq V(t)$, then $V\left(t s^{-1}\right) \leqq V(1)$, so $t \in s A$. Hence $t^{2 n} \in s^{n} t^{n} A \subseteq M$ which proves that $t \in N$.

Following [2, Definition 1, p. 134] we say two valuation pairs $\left(A_{1}, P_{1}\right)$ and $\left(A_{2}, P_{2}\right)$ of $R$ are dependent if the ring generated by $A_{1}$ and $A_{2}$ is properly contained in $R$. Since $R$ has few zero divisors, this is equivalent to the existence of a valuation pair $(A, P)$ of $R$ such that for $i=1,2, A \supseteq A_{i} \supseteq P_{i} \supseteq P \supseteq V_{i}^{-1}(0)$ where $V_{i}$ is the valuation associated with $\left(A_{i}, P_{i}\right)$. This second form of this definition is the one used by Manis [6].

TheOREM 4. Let $\left\{\left(A_{i}, P_{i}\right)\right\}_{i=1, \cdots, n}$ be pairwise independent valuation pairs of $R$ with valuations $V_{i}$ and groups $\Gamma_{i} \backslash\left\{V_{i}(0)\right\}$. For $x_{1}, x_{2}$, $\cdots, x_{n} \in R$ and $\alpha_{i} \in \Gamma_{i} \backslash\left\{V_{i}(0)\right\}$ for $i=1, \cdots, n$, there exists $x \in R$ such that $V_{i}\left(x-x_{i}\right) \leqq \alpha_{i}$ for $i=1, \cdots, n$.

Proof. We may assume that each $V_{i}$ is proper. Let $A=\bigcap_{i=1}^{n} A_{i}$ and $Q_{i}=A \cap P_{i}$. Since the total quotient ring of $A$ is $R$, there exist $a, s, a_{1}$, $\cdots, a_{n} \in A, s$ regular, such that $x_{i}=a_{i} / s$ and $x=a / s$. Then $V_{i}\left(a-a_{i}\right)$ $\leqq \alpha_{i} V_{i}(s)$ for all $i$ will imply the result. Hence we may assume that $x_{1}, \cdots, x_{n} \in A$. Also we may assume that $\alpha_{i}<V_{i}(1)$ for all $i$. Let $M_{i}=\left\{z \in R \mid V_{i}(z) \leqq \alpha_{i}\right\}$ and set $N_{i}=M_{i} \cap A$. For $x \in A, V_{i}\left(x-x_{i}\right) \leqq \alpha_{i}$ is equivalent to $x \equiv x_{i}\left(\bmod N_{i}\right)$. Thus it suffices to show that the canonical map $A \rightarrow \prod_{i=1}^{n} A / N_{i}$ is onto; i.e., that the $N_{i}$ are pairwise 
comaximal [1, Proposition 5, p. 72]. By Theorem 3, $Q_{1}, \cdots, Q_{n}$ are the maximal regular ideals of $A$. Thus it suffices to show that $N_{i} \Phi Q_{j}$ for $i \neq j$.

Suppose that $N_{i} \subseteq Q_{j}$ for some $i \neq j$. Using Lemma 2 one sees that $N=\sqrt{ } N_{i}$ is a prime ideal of $A$ so that $N \subseteq Q_{j}$. Also since $\alpha_{i}<V_{i}(1)$, $N_{i} \subseteq Q_{i}$ so that $N \subseteq Q_{i}$. Hence $A_{i}=A_{S\left(Q_{i}\right)} \subseteq A_{S(N)}$ and similarly $A_{j} \subseteq A_{S(N)}$. Each $M_{i}$ contains a regular element, for let $u \in R$ be such that $V_{i}(u)=\alpha_{i}$. Then $V_{i}(w)=\alpha_{i}^{-1}$ for some $w \in R$, say $w=x / y$ with $x, y \in A, y$ regular. Let $p$ be a regular element of $P_{i}$. Then $w u \in A_{i} \backslash P_{i}$ and $w y p \in P_{i}$ showing that $V_{i}(y p)<\alpha_{i}$; i.e., that $y p \in M_{i}$. By $[1$, Proposition 10, p. 89] $M_{i}=N_{i} A_{S\left(Q_{i}\right)}$, so $N_{i}$ must contain a regular element; therefore $N$ does too and hence $A_{S(N)} \neq R$. This contradicts the independence of $A_{i}$ and $A_{j}{ }^{2}$

\section{REFERENCES}

1. N. Bourbaki, Algèbre commutative, Chapters 1 and 2, Actualités Sci. Indust., No. 1290, Hermann, Paris, 1961.

2. - Algèbre commutative, Chapters 5 and 6, Actualités Sci. Indust., No. 1380, Hermann, Paris, 1964.

3. E. D. Davis, Overrings of commutative rings. II. Integrally closed overrings, Trans. Amer. Math. Soc. 110 (1964), 196-212.

4. D. K. Harrison, Finite and infinite primes for rings and fields, Mem. Amer. Math. Soc., No. 68, (1966).

5. Max D. Larsen, Some containment relations between classes of regular ideals in a ring with few zero divisors, J. Sci. Hiroshima Univ. Ser. Math. A-I 32 (1968), 241-246.

6. Merle Manis, Valuations on a commutative ring, $\mathrm{Ph} . \mathrm{D}$. Thesis, Univ. of Oregon, Eugene, 1966.

\section{UNIVERSITY OF NEBRASKA}

2 The author has learned that Malcolm Griffin has obtained Remark 4 and Theorem 2 independently in a paper to appear in J. Reine Angew. Math. 